\title{
Mixed Incontinence: Does Preoperative Urodynamic Detrusor Overactivity Affect Postoperative Quality of Life After Pubovaginal Sling?
}

\author{
John T. Stoffel, John J. Smith, Simone Crivellaro, John F. Bresette \\ Department of Urology (JTS, JFB), Lahey Clinic, Burlington, Massachusetts, USA and Department \\ of Urology (JJS, SC), Wake Forest, South Carolina, USA
}

\begin{abstract}
Objective: Our purpose was to determine if women with mixed urinary incontinence (MUI) and urodynamic detrusor overactivity (DO) have less improvement in urinary symptoms after pubovaginal sling surgery (PVS), compared to MUI without DO.

Materials and Methods: Women with preoperative MUI symptoms prior to PVS were identified through retrospective review. DO was defined as a symptomatic $5 \mathrm{~cm} \mathrm{H}_{2} 0$ detrusor pressure or greater rise during urodynamics. MUI patients with and without DO before PVS were divided into Groups A and B, respectively. All patients had returned a completed Urogenital Distress Inventory 6 (UDI-6) questionnaire and a 3-day diary of pad usage before surgery and at each postoperative visit. Study endpoints included change in total UDI-6 score, and change in number of pad use/day after PVS.

Results: 73 patients were identified, 31 in Group A and 42 in Group B. Mean follow-up after PVS was 15 and 16 months, respectively $(\mathrm{p}=0.59)$. Preoperative total UDI-6 scores were 11.8 and $12.7(\mathrm{p}=0.30)$ for Group A and B. Mean changes in total UDI-6 after PVS were - 8.0 and - $10.2(\mathrm{p}=0.030)$, respectively. After PVS, both groups reported similar mean reduction in $\mathrm{pad} /$ day usage from preoperative baseline $(-2.57 \mathrm{vs} .--2.49, \mathrm{p}=0.83)$. There were no differences between the groups when comparing demographic, urodynamic, or operative data.

Conclusion: MUI patients had improved continence and quality of life after PVS. However, MUI patients with DO had less improvement in UDI-6 scores after PVS, despite a similar reduction to pad use/day.
\end{abstract}

Key words: urodynamics; urinary incontinence; urge incontinence; suburethral slings

Int Braz J Urol. 2008; 34: 765-71

\section{INTRODUCTION}

Mixed urinary incontinence (MUI) is clinically defined by as "involuntary leakage associated with urgency and also with exertion" (1). In theory, the urge incontinence component of this definition is likely caused by an uninhibited bladder contraction. During urodynamic studies, uninhibited contractions, defined as Detrusor Overactivity (DO), are identified through symptomatic rises in the detrusor pressure during the study filling phase (2). Interestingly, many women with clinically defined MUI do not demonstrate DO during urodynamic studies (3). Consequently, the relationship between DO and clinical symptoms is often conflicting and has not been fully delineated.

Many women with symptomatic MUI are offered surgical therapy. However, surgical cure rates 
for the MUI patient are highly variable and range from 60 - 97\% across several different surgical techniques including retropubic suspensions $(4,5)$, pubovaginal slings (PVS) $(6,7)$, and tension free procedures (8). Currently, it is not fully known if pre-operative urodynamic data can be used to stratify surgical risk for MUI patients undergoing incontinence surgery. Consequently, our purpose was to determine if women with clinically diagnosed mixed urinary incontinence (MUI) and urodynamic detrusor overactivity have less improvement in urinary symptoms after pubovaginal sling surgery (PVS), compared to MUI without urodynamic detrusor overactivity.

\section{MATERIALS AND METHODS}

Women treated with pubovaginal slings between June 1998 and April 2005 were retrospectively identified from surgical case logs. For this study, the International Continence Society (ICS) definition of MUI was used, as cited above. For data extraction purposes, the definition was contracted to a documented clinical history of both stress and urge incontinence symptoms occurring more than once a week. For patients meeting these criteria, charts were reviewed for demographic, physical exam, urodynamic, surgical, and post operative data.

Prior to surgery, all patients had been evaluated with a Laborie Aquarius (Williston, VT) multichannel urodynamic system synchronized with fluoroscopic imaging. Studies were performed using International Continence Society good urodynamic technique recommendations. All patients had been screened for unrecognized urinary tract infections prior to testing. Testing was performed in the standing/upright position with bladder/ rectal air charged catheters. The urodynamic protocol included standardized filling rates of $55 \mathrm{cc} /$ minute with Renografin (Bracco Diagnostics Inc, Princeton NJ) until the feeling of strong urge, an uninhibited detrusor contraction causing incontinence, or a $400 \mathrm{cc}$ limit. Valsalva leak point pressures were measured at least twice at volumes correlating to feelings of strong urge or 400 cc. If urge incontinence occurred prior to Valsalva testing, the detrusor pressures were allowed to nadir and the bladder was refilled to the highest previously recorded volume and Valsalva testing was performed. All pressure/flow studies utilized intubated flow rates. Fluoroscopic images in the anterior-posterior and lateral positions were taken regularly during the study and were correlated to the cystometrogram tracings. All studies were performed by an experienced urogynecology nurse trained in urodynamic testing.

Detrusor overactivity (DO) was defined as any $5 \mathrm{~cm} \mathrm{H}_{2} 0$ symptomatic involuntary rise in detrusor pressure during the testing, per ICS recommendations. For this study, no differentiation was made between spontaneous or provoked DO. The number of contractions and maximum contraction amplitude was not recorded. All detrusor overactivity was considered idiopathic unless a relevant neurologic condition was present. Based on these definitions, patients were divided into two groups, those with urodynamic proven detrusor overactivity (Group A) and those without overactivity (Group B).

All patients had been treated with a cadaveric dermis bladder neck sling (9) or autologous rectus fascia bladder neck sling suspended over the rectus fascia. Operative data, including operative time, blood loss, and complications, were recorded. Post operative retention, defined as clean intermittent catheterization post void residual greater than $150 \mathrm{cc}$, was documented. Post operative care followed a specific protocol including instructions for quantifying the number of incontinence pads used per day starting 72 hours prior to a scheduled follow up visit. All patients were scheduled for at least 3 routine follow up visits during the first 12 months after surgery and then at 6 to 12 month intervals afterwards. Incontinence pad type was not standardized in this retrospective study.

At each visit, both before and after PVS, a registered urogynecology nurse solicited information on patient's current pad usage and all patients completed a validated quality of life questionnaire, the Urogenital Distress Inventory 6 (UDI-6). This questionnaire is a robust 6 domain validated urinary incontinence specific questionnaire that measures distress caused by multiple urinary symptoms, including urgency, urge incontinence, stress incontinence, urinary retention, and pelvic pain (10).

End points used in this study included change in pad use/day and change in UDI scores after PVS surgery. The change in total UDI score after PVS and 
change in pad use/day was calculated for each subject using data available from each patient's initial and last follow-up visit. Continuous variables were compared using Student-t-Tests or Wilcoxon sum rank tests. Binomial variables were compared with Chi-Square tests. Tests with $\mathrm{p}<0.05$ were considered statistically significant.

\section{RESULTS}

A total 262 patient underwent PVS between 1998 and 2005. Seventy-three met the inclusion criteria for pre-operative MUI prior to PVS. Cadaveric dermis was utilized in 70 patients and rectus fascia used in 3. Of the 73 patients, 31 had preoperative urodynamic overactivity (Group A), all of which were classified as idiopathic, and 42 did not have urodynamic overactivity (Group B). Two patients in Group A were treated with rectus fascia and 1 in Group B. One patient in Group B had an L5 radiculopathy, but demonstrated no urodynamic changes. Mean follow-up after PVS for Groups A and B were 16 and 15 months (range $1-24, p=0.59$ ).

Prior to PVS, 7 patients in Group A and 9 in Group B were taking anticholinergic medications. Four patients in Group A and 7 in Group B had pre- vious urethral bulking agent treatment. Vaginal vault prolapse was a common comorbidity, although both groups showed similar degrees of prolapse along the anterior wall (2.7 vs. 2.5 Baden Walker Grade, p = 0.56 ), apical (1.0 vs. $1.2 \mathrm{BW}, \mathrm{p}=0.75)$, and posterior wall (1.3 vs. 1.0 BW, p = 0.47). Pre-operative urodynamic characteristics were likewise similar, with comparable flow rates (20 and $23 \mathrm{~mL} / \mathrm{s}, \mathrm{p}=0.36$ ), Valsalva leak point pressures (60 and $74 \mathrm{~cm} \mathrm{H}_{2} 0$, p $=0.160)$, and post void residuals $(13$ and $31 \mathrm{~mL}, \mathrm{p}$ $=0.114)$. After PVS, Groups A and B reported 2.5 and 5.2 days of urinary retention $(p=0.0002)$. There were no other significant differences between the two groups when comparing age, parity, menopause status, concomitant vaginal vault prolapse, or surgical blood loss, summarized in Table-1.

Groups A and B had similar pre-operative UDI scores (Figure-1). After PVS, mean changes in total UDI-6 scores were - 8.0 and $-10.2(\mathrm{p}=0.030)$, respectively. The study was not adequately powered to perform a meaningful sub-group analysis of the UDI-6 domains. Prior to surgery, both groups also had similar pad use/day (Figure-2). After PVS mean change in pad use/day was -2.57 and $2.49(p=0.64)$ for Groups A and B, respectively. After PVS, 21 (68\%) and $28(67 \%)$ patients in Group A and B, respectively, did not wear pads for protection $(p=0.92)$. A total of

Table 1 - Patient demographics.

\begin{tabular}{|c|c|c|c|c|}
\hline & & $\begin{array}{c}\text { Group A } \\
\text { (+ Overactivity) }\end{array}$ & $\begin{array}{c}\text { Group B } \\
\text { (- Overactivity) }\end{array}$ & p Value \\
\hline Mean age & & 57.6 years (SD 12.6) & 58.6 years (SD 12.1) & 0.69 \\
\hline Mean parity & & 2.6 births (SD 1.7) & 2.4 births (SD 1.6) & 0.47 \\
\hline Hysterectomy & Yes & 16 patients & 16 patients & 0.62 \\
\hline Prolapse & Yes & 25 patients & 35 patients & 0.77 \\
\hline Menopause & Post & 25 patients & 35 patients & 0.77 \\
\hline Pre op anticholinergic & & 7 patients & 9 patients & 0.90 \\
\hline Pre op VLPP & & $60 \mathrm{~cm} \mathrm{H}_{2} 0(\mathrm{SD} 27)$ & $74 \mathrm{~cm} \mathrm{H}_{2} 0(\mathrm{SD} 39)$ & 0.16 \\
\hline Pre op flow rate & & 20 cc/s (SD 12) & 23 cc/s (SD 17) & 0.36 \\
\hline Bladder capacity & & 330 cc (SD 93) & $355 \mathrm{cc}$ (SD 143) & 0.40 \\
\hline Mean blood loss & & 213 cc (SD 142) & 273 cc (SD 159) & 0.10 \\
\hline Post op retention & & 2.5 days (SD 2.2) & 5.2 days (SD 3.3) & 0.0002 \\
\hline
\end{tabular}




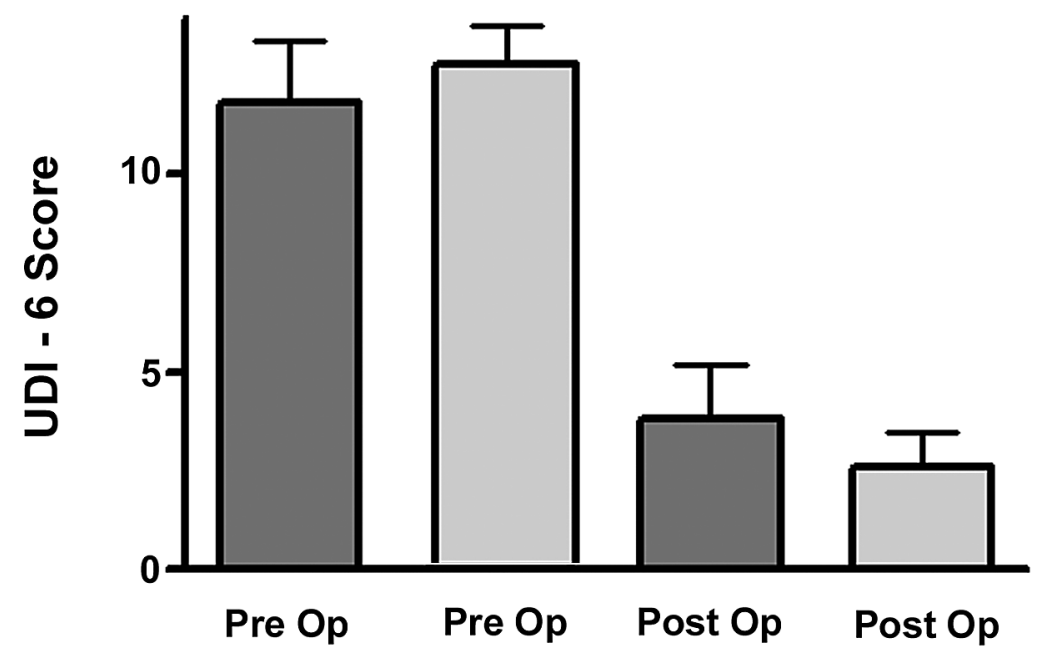

Figure 1 - Mean UDI-6 scores before and after PVS. Group A = dark grey bars; Group B = light grey bars.

6 patients, 3 within each group required either a collagen injection or sling revision for persistent stress incontinence. For the 16 patients using anticholingeric medication before surgery, all continued to use the medication after PVS. No new patients were using anticholinergics at the last post operative visit.

\section{COMMENTS}

Risk factors that influence surgical outcomes in the MUI population are not well described or understood. Our study investigated whether MUI patients with DO were at risk for worse outcomes

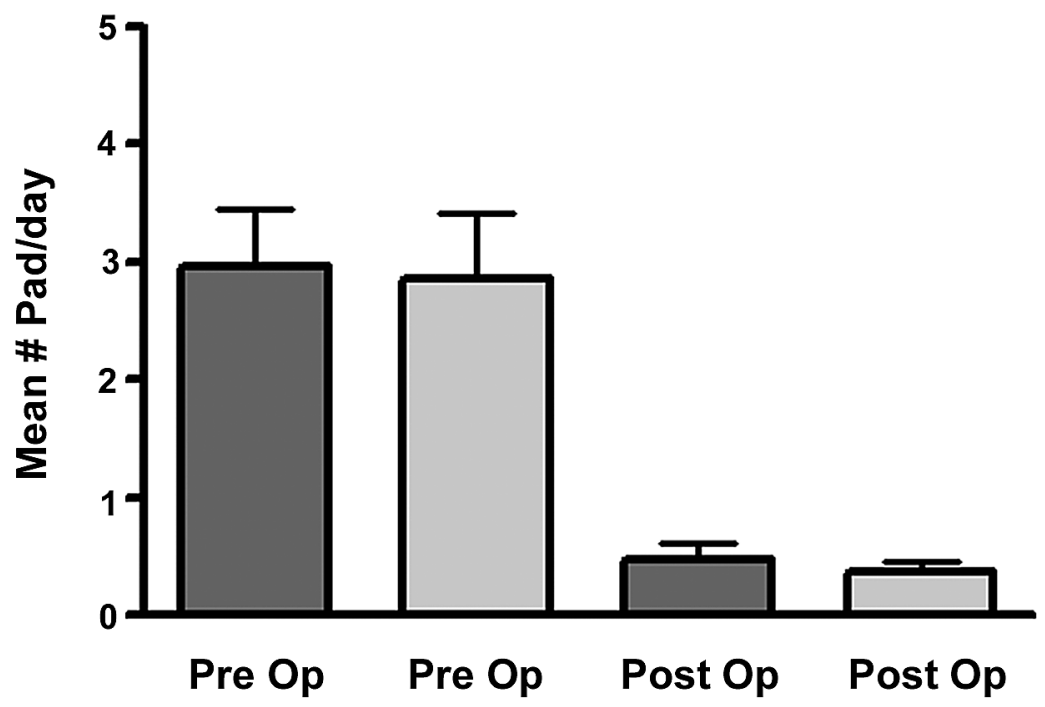

Figure 2 - Mean pad use per day. Group A = dark grey bars; Group B = light grey bars. 
after PVS, compared to MUI patients without DO. We demonstrated that while both groups showed similar improvement in pad use/day, MUI patients with preoperative DO had significantly less improvement in self reported urinary specific symptoms after PVS, as measured by the Urogenital Distress 6 quality of life questionnaire.

There is conflicting information regarding the impact of pre-operative urodynamic overactivity on surgical outcomes in the MUI populations. Kuzmarov studied 51 women with MUI prior to Marshall Marchetti Kranz (MMK) suspension and observed no relationship between detrusor overactivity and outcome (11). Del Campo Rodriguez found no difference in cure rates after Burch or MMK for 44 women with pre-existing urodynamic overactivity (12). Miller et al. also did not find an association between pre-operative detrusor overactivity and return of bladder function (13). However, Paick et al. recently reported that MUI patients with uninhibited detrusor contractions during cystometry should be considered a high risk group for surgical failure following tension-free vaginal tape, suprapubic arc sling, or transobturator tape treatments (14). Depending on the endpoints used, our study supports both sides of these contradictory series. In our study we noted no difference between groups using the endpoint pad usage/day but noted a significant different when comparing health related quality of life (HRQOL) scores. It is possible that our study was underpowered to detect a meaningful change between a weak variable, such as pad usage/day, but adequately powered to detect a difference when using a strong validated variable, such as the UDI-6 total score. Consequently, discrepancies in the literature regarding the relationships between MUI, DO, and surgical outcomes may be attributed to wide variation in study endpoints. More research with standardized endpoints is clearly needed in this area before these relationships can be better understood.

It is unlikely that patient demographics greatly influenced our findings. In general, our patient sample represented a typical MUI population seeking treatment. In our study of 73 women, preoperative DO was identified in $42 \%$. These findings are similar to the $43 \%$ prevalence found in much a larger series of 1626 women with MUI (15). Furthermore, the Valsalva leak point pressures and prevalence of vaginal vault prolapse found within our patient sample are similar to other MUI surgical series $(6,13-15)$. Within the study, women with and without DO also appeared to have similar demographics. Stress incontinence intensity may have been a confounding factor, although this is unlikely since groups had similar mean pre-operative UDI-6 scores and Valsalva leak points.

Likewise, it is unlikely that findings in this study can be attributed to the UDI-6 instrument. The UDI-6 QOL questionnaire used in this study is a well validated, sensitive, and specific urinary symptom specific instrument. The questionnaire has 6 domains, including urinary urgency, urge incontinence, stress incontinence, urinary retention, and pelvic pain and has been reliably validated across patient age and diagnosis. Although total UDI-6 score has not been routinely used to assess outcomes after MUI surgery, it is commonly used to determine outcomes for overactive bladder treatment (16) and effectiveness for other anti incontinence interventions (17). Future investigations with other validated quality of life (QOL) questionnaires should determine if the results generated by the UDI-6 in this study are questionnaire specific.

However, a limitation of this study is that MUI was considered a binomial variable and patients were stratified into those with DO (Group A) and those without (Group B). Since voiding diaries were not available, we did not sub-stratify Group A or Group B by number of urge incontinence episodes either before or after surgery. Given the small sample size, we also chose not to sub-stratify DO in Group A by number of contractions or amplitude of contraction. Consequently, it is possible that the intensity of urgency symptoms is a confounding factor in our findings. With a larger sample size, we may have confirmed Kulseng-Hanssen's recently published findings that MUI patients with urge predominant symptoms may experience a lower QOL after TVT, compared to MUI patients with stress predominant symptoms (18). A larger sample size may have better discerned the influence of symptom intensity on MUI surgical outcome.

A further limitation of this and of all urodynamic based MUI investigations is that urodynamic protocols vary widely from center to center. In 2003, Sriram et al. performed an audit of United Kingdom 
urodynamic practitioners and found considerable disagreement among standardization of catheter zeroing techniques and application of urodynamic definitions (19). Verbal instructions given to patients during testing have also been shown influence the detection of idiopathic detrusor overactivity in patients with clinical symptoms of urinary urgency (20). Although we acknowledge the difficulty in reproducing urodynamic data, we attempted to minimize patient to patient variability by adhering to a written urodynamic protocol and having a single practitioner perform all studies. However, we do acknowledge inter-institutional protocol variability as a further potential confounding factor in this study.

Finally, we recognize the limitations surrounding the retrospective design of this study. Although our patient population represents an unselected group of women presenting for evaluation and treatment of mixed incontinence, unrealized confounding variables may bias our retrospective data extraction. A prospective, multi-center study would better minimize potential bias.

\section{CONCLUSION}

PVS for treatment of MUI is associated with an improvement in pad usage/day and UDI-6 total scores. However, MUI patients with DO have less improvement in UDI-6 scores after PVS, compared to MUI patients without DO. Preoperative urodynamic testing, in combination with HR-QOL questionnaire administration, should be considered as a pre-operative tool for addressing post operative QOL expectations after PVS for this patient population.

\section{CONFLICT OF INTEREST}

None declared.

\section{REFERENCES}

1. Abrams P, Cardozo L, Fall M, Griffiths D, Rosier P, Ulmsten $\mathrm{U}$, et al.: The standardisation of terminol- ogy in lower urinary tract function: report from the standardisation sub-committee of the International Continence Society. Urology. 2003; 61: 37-49.

2. Weber AM, Abrams P, Brubaker L, Cundiff G, Davis G, Dmochowski RR, et al.: The standardization of terminology for researchers in female pelvic floor disorders. Int Urogynecol J Pelvic Floor Dysfunct. 2001; 12: 178-86.

3. McGuire EJ: Mixed symptomatology. BJU Int. 2000; 85 (Suppl 3): 47-52; discussion 55-6.

4. Karram MM, Bhatia NN: Management of coexistent stress and urge urinary incontinence. Obstet Gynecol. 1989; 73: 4-7.

5. Colombo M, Zanetta G, Vitobello D, Milani R: The Burch colposuspension for women with and without detrusor overactivity. Br J Obstet Gynaecol. 1996; 103: 255-60.

6. Chou EC, Flisser AJ, Panagopoulos G, Blaivas JG: Effective treatment for mixed urinary incontinence with a pubovaginal sling. J Urol. 2003; 170: 494-7.

7. Osman T: Stress incontinence surgery for patients presenting with mixed incontinence and a normal cystometrogram. BJU Int. 2003; 92: 964-8.

8. Holmgren C, Nilsson S, Lanner L, Hellberg D: Longterm results with tension-free vaginal tape on mixed and stress urinary incontinence. Obstet Gynecol. 2005; 106: 38-43.

9. Crivellaro S, Smith JJ, Kocjancic E, Bresette JF: Transvaginal sling using acellular human dermal allograft: safety and efficacy in 253 patients. J Urol. 2004; 172: 1374-8.

10. Uebersax JS, Wyman JF, Shumaker SA, McClish DK, Fantl JA: Short forms to assess life quality and symptom distress for urinary incontinence in women: the Incontinence Impact Questionnaire and the Urogenital Distress Inventory. Continence Program for Women Research Group. Neurourol Urodyn. 1995; 14: 1319.

11. Kuzmarov IW: Urodynamic assessment and chain cystogram in women with stress urinary incontinence. Clinical significance of detrusor instability. Urology. 1984; 24: 236-8.

12. del Campo-Rodríguez M, Batista-Miranda JE, Errando-Smet C, Arañó-Bertrán P: Outcome of colposuspension in patients with stress urinary incontinence and abnormal cystometry. Arch Esp Urol. 1999; 52: 810-4.

13. Miller EA, Amundsen CL, Toh KL, Flynn BJ, Webster GD: Preoperative urodynamic evaluation may predict voiding dysfunction in women undergoing pubovaginal sling. J Urol. 2003; 169: 2234-7. 
14. Paick JS, Oh SJ, Kim SW, Ku JH: Tension-free vaginal tape, suprapubic arc sling, and transobturator tape in the treatment of mixed urinary incontinence in women. Int Urogynecol J Pelvic Floor Dysfunct. 2008; 19: 123-9.

15. Digesu GA, Salvatore S, Fernando R, Khullar V: Mixed urinary symptoms: What are the urodynamic findings? Neurourol Urodyn. 2008; 27: 372-5.

16. van der Vaart CH, de Leeuw JR, Roovers JP, Heintz AP: Measuring health-related quality of life in women with urogenital dysfunction: the urogenital distress inventory and incontinence impact questionnaire revisited. Neurourol Urodyn. 2003; 22: 97-104.

17. Woodman PJ, Misko CA, Fischer JR: The use of short-form quality of life questionnaires to measure the impact of imipramine on women with urge incontinence. Int Urogynecol J Pelvic Floor Dysfunct. 2001; 12: 312-5; discussion 315-6.

18. Kulseng-Hanssen S, Husby H, Schiotz HA: The tension free vaginal tape operation for women with mixed incontinence: Do preoperative variables predict the outcome? Neurourol Urodyn. 2007; 26: 115-21; discussion 122 .

\section{EDITORIAL COMMENT}

This is a nice paper by Stoffel et al. looking at one of the most difficult group of women we consider doing the sling surgery on: women with mixed urinary incontinence (MUI) and urodynamic detrusor overactivity (DO). The authors reviewed 73 women with preoperative MUI symptoms prior to sling surgery. MUI patients with and without DO were divided into two groups and followed-up with a questionnaire and pad test after the sling surgery.

Of the 73 patients, 31 women had DO and 42 did not. After surgery, both groups reported similar mean reduction in pad/day usage but MUI women with DO had less improvement in validated incontinence questionnaire despite a similar reduction if pad use/day. The UDI-6 QOL questionnaire used in this study is a well validated, sensitive, and specific urinary symptom specific instrument. However, a limitation of this study is that MUI was considered a binomial variable and patients were stratified into those with DO and those without. Voiding diaries
19. Sriram R, Ojha H, Farrar DJ: An audit of urodynamic standardization in the West Midlands, UK. BJU Int. 2002; 90: 537-9.

20. Blaivas JG, Groutz A, Verhaaren M: Does the method of cystometry affect the incidence of involuntary detrusor contractions? A prospective randomized urodynamic study. Neurourol Urodyn. 2001; 20: 141-5.

Accepted after revision: July 28, 2008

\section{Correspondence address:}

Dr. John T. Stoffel

Department of Urology

Lahey Clinic

41 Mall Road

Burlington, MA, 01805, USA

Fax: + 1781 744-5429

E-mail: John.T.Stoffel@1ahey.org

were not available so a key parameter of number of urges incontinence episodes either before or after surgery were not available.

There are good data in this paper as the authors correctly pointed out that the sling operation for MUI is associated with an improvement in pad usage/day and symptom index. However, MUI patients with DO have less improvement in UDI-6 scores compared to MUI patients without DO.

Dr. Michael B. Chancellor Department of Urology William Beaumont Hospital Royal Oak, Michigan, USA E-mail: chancellormb@gmail.com 